\title{
Also set-valued functions do not like iterative roots
}

\author{
Witold Jarczyk and Weinian Zhang \\ Witold Jarczyk obtained his $\mathrm{PhD}$ and habilitation from the Silesian University in 1983 \\ and 1993, respectively. Presently, he is professor of mathematics at the University \\ of Zielona Góra in Poland. His interests lie in functional equations and inequalities, \\ iteration theory and discrete dynamical systems. \\ Weinian Zhang received his PhD from the Peking University in 1990. Presently, he \\ is professor of mathematics at the Sichuan University in China. His interests lie in \\ bifurcation theory of differential equations, iteration theory and dynamical systems.
}

\section{Introduction}

It seems that it is Ch. Babbage who first, yet at the beginning of the 19th century, wrote on iterative roots explicitly. Given a mapping $f: X \rightarrow X$ and a positive integer $n \geq 2$, the problem is to find a mapping $g: X \rightarrow X$ such that the $n$-th iterate of $g$, the composition $g^{n}$ of $n$ copies of $g$, is $f$, i.e., to solve the functional equation

$$
g^{n}=f
$$

In [1] Babbage studied (1.1) for $f$ being the identity mapping. After him a lot of results concerning the general case of (1.1) in various settings have been proved. Many of them can be found in the monographs [12] and [13] by M. Kuczma and M. Kuczma, B. Choczewski, R. Ger, respectively, as well as in the book [19] by Gy. Targonski. Some recent results have been presented in the survey papers [3] and [2].

Die Aufgabe, die $n$-te iterative Wurzel einer Abbildung $f: X \rightarrow X$ zu finden, besteht darin, eine Funktion $g: X \rightarrow X$ so zu bestimmen, dass $g^{n}=g \circ g \circ \ldots \circ g=f$ ( $n$-fache Hintereinanderausführung) gilt. Für dieses Problem sind sowohl kombinatorische, als auch analytische Resultate bekannt. So besitzt beispielsweise $f:[0,1] \rightarrow[0,1]$, gegeben durch $f(x)=4 x(1-x)$, keine iterative Wurzel. Die Autoren untersuchen in dieser Arbeit das analoge Problem für mengenwertige Abbildungen $f: X \rightarrow 2^{X}$. Es zeigt sich, dass selbst Monotonie- und Stetigkeitsannahmen, die bei gewöhnlichen Funktionen Existenz von Wurzeln sicher stellen, hierfür in diesem Fall im allgemeinen nicht ausreichen. 
The purely combinatorial paper [10] by R. Isaacs gave a description of solutions to (1.1) for an arbitrary bijection $f$. The case of general $f$ was completely solved by G. Zimmermann, Ph.D. student of Targonski, in her not well-known doctoral thesis [22] (see also [17] by G. Riggert noticing that Zimmermann is the maiden name of Riggert).

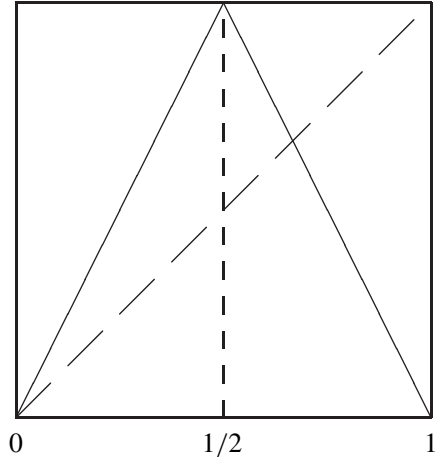

Fig. 1: Hat function

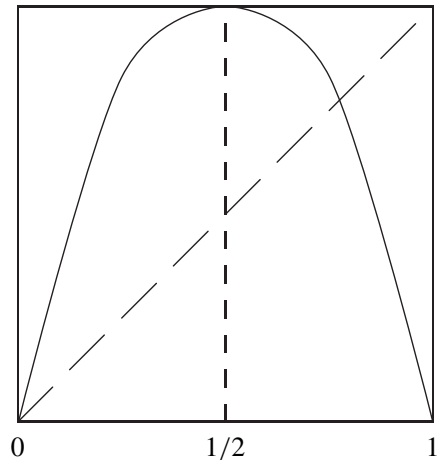

Fig. 2: Parabola $y=4 x(1-x)$

It turns out that even very simple and nice functions can have no roots. For instance, this is the case if $f$ is the so-called hat function, i.e. $f(x)=\min \{2 x, 2-2 x\}$ for $x \in[0,1]$ (see Fig. 1) or $f$ is the celebrated parabola $y=4 x(1-x)$ for $x \in[0,1]$ (see Fig. 2). Of course, lack of roots for these functions can be deduced from Zimmermann's work. However, the reader surely can give a short and quite elementary argument in both cases. The above mentioned functions represent two important classes of mappings: piecewise monotone functions and polynomials. As follows, from [5], even in the class of piecewise monotone functions equation (1.1) leads to non-trivial questions. For $f$ in this class sufficient conditions for nonexistence and existence of roots can be found in [21]. For polynomials the lack of roots is also a rather common phenomenon. A fundamental paper is [16] by R.E. Rice, B. Schweizer and A. Sklar, published in the Monthly almost 25 years ago. The answer to its title question "When is $f(f(z))=a z^{2}+b z+c$ ?" is never. Similar results concerning some other polynomials can be found in [7] and [8]. Nonexistence of roots, both formal and holomorphic, was indicated by S. Bogatyi in his important article [6].

Difficulties appearing when solving equation (1.1), even in the class of continuous monotone self-mappings of an interval, have been enlightened in the crucial paper [9] by P.D. Humke and M. Laczkovich. Roughly speaking, they proved that the set of functions having a root is an analytic but non-Borel subset of the space $C([0,1], \mathbb{R})$ endowed with the sup-norm. The papers [18] and [4] by K. Simon and A. Blokh, respectively, show that this set is small in $C([0,1],[0,1])$ both from the category (see $[18,4])$ and measuretheoretical (cf. [18]) points of view. Nonexistence of roots is typical also for some regular functions (see [20]).

Recently some natural ideas of using set-valued functions have been examined (cf., for instance, $[14,15,11])$. One can consider replacing single-valued functions by set-valued functions in (1.1) both for $f$ and $g$. It seems that up to now there are no notions leading to a satisfactory result in such a case. 
In this paper we show that the phenomenon of lack of iterative roots appears also when studying some set-valued functions with exactly one value not being a singleton. Even imposing assumptions like continuity or strict monotonicity on the "single-valued parts" of such a set-valued function does not guarantee the existence of its square roots (see Example 2). This shows that maybe the situation for set-valued functions is even more sophisticated since those assumptions usually allow us to find roots in the single-valued case.

\section{Main results}

Given a set-valued function $f: X \rightarrow 2^{Y}$, the image $f(A)$ of a set $A \subset X$ is defined by

$$
f(A)=\bigcup_{x \in A} f(x)
$$

Then we can introduce the composition $g \circ f$ of set-valued functions $f: X \rightarrow 2^{Y}$ and $g: Y \rightarrow 2^{Z}$ by the familiar formula

$$
(g \circ f)(x)=g(f(x)) .
$$

Clearly this operation is associative. So, for every positive integer $n$, we can define the $n$-th iterate of $g: X \rightarrow 2^{X}$ as the composition of $n$ copies of $g$ :

$$
g^{n}=\underbrace{g \circ \ldots \circ g}_{n-\text { times }} .
$$

Consequently, the problem of looking for solutions $g: X \rightarrow 2^{X}$ to (1.1) for set-valued functions $f$ is posed in a proper way.

Remark that if $g: X \rightarrow 2^{X}$ is an iterative root of $f: X \rightarrow 2^{X}$ then $f$ and $g$ commute, i.e. $f \circ g=g \circ f$. In fact, assume that $g^{k}=f$ for a positive integer $k$ and fix an $x \in X$. If $z \in f(g(x))$ then $z \in f(y)$ for a $y \in g(x)$, that is, $z \in g^{k}(g(x))=g\left(g^{k}(x)\right)=g(f(x))$. Conversely, if $z \in g(f(x))$ then $z \in g(y)$ for a $y \in f(x)$, so $z \in g(f(x))=g\left(g^{k}(x)\right)=$ $g^{k}(g(x))=f(g(x))$.

In what follows, we consider $X$ as an arbitrary set and let $\# A$ denote the cardinality of a subset $A \subset X$.

Proposition. Consider a set-valued function $f: X \rightarrow 2^{X}$ and let $g: X \rightarrow 2^{X}$ be its iterative square root. If there is a point $c \in X$ such that

(i) \#f(x)=1 for every $x \in X \backslash\{c\}$ and

(ii) $f\left(x_{0}\right)=\{c\}$ for an $x_{0} \in X$,

then $\# g(c) \leq 1$.

Proof. Suppose that

$$
\# g(c) \geq 2 \text {. }
$$

It follows from (i) that $g$ has non-void values only. Fix a $p \in g\left(x_{0}\right)$. Then

$$
g(p) \subset g\left(g\left(x_{0}\right)\right)=f\left(x_{0}\right)=\{c\},
$$


that is in fact $g(p)=\{c\}$, whence

$$
f(p)=g(g(p))=g(c) .
$$

Therefore, by (2.2) and (i), we get $p=c$. Thus, we have proved that $g\left(x_{0}\right)=\{c\}$, which gives

$$
g(c)=g^{2}\left(x_{0}\right)=f\left(x_{0}\right)=\{c\}
$$

a contradiction to (2.2).

Our main results are simple consequences of the proposition.

Theorem 1. Let $f: X \rightarrow 2^{X}$. If there are a point $c \in X$ and a positive integer $n$ such that (i) and (ii) hold,

(iii) $\# f(c)>n$, and

(iv) $\#\{x \in X: f(x)=\{y\}\} \leq n$ for every $y \in X$,

then $f$ has no iterative square roots.

Proof. Suppose that $f$ has a square root $g: X \rightarrow 2^{X}$. By (i) and (iii) all the values of $f$ and consequently of $g$ are non-void.

Firstly, we claim that

$$
\# g(x) \leq n \quad \text { for } \quad x \in X \backslash\{c\}
$$

In order to see this, fix an $x \in X \backslash\{c\}$. Take any $v \in g(x)$. Since $f(x)$ is a singleton and

$$
f(x)=g(g(x))=\bigcup_{u \in g(x)} g(u),
$$

for every $u \in g(x)$ we have $g(u)=g(v)$, whence $f(u)=f(v)$. This gives the inclusion

$$
g(x) \subset\{u \in X: f(u)=f(v)\} .
$$

If $g(x)$ is not a singleton then, according to (2.4) and (i), $f(v)$ is a singleton, whence using (2.4) again and (iv) we complete the proof of (2.3).

Since all the values of $g$ are non-void, it follows from the proposition that $g(c)=\{u\}$ with a $u \in X$. Then $g(u)=g^{2}(c)=f(c)$, whence, by (iii), we have \#g(u)>n. So, $u \neq c$, which contradicts $(2.3)$.

Theorem 2. Let $f: X \rightarrow 2^{X}$. If there is a point $c \in X$ such that (i) and (ii) hold,

(v) $\# f(c)>1$, and

(vi) $c \in f(c)$,

then $f$ has no iterative square roots.

Proof. Suppose that $f$ has a square root $g: X \rightarrow 2^{X}$. By (i) and (v) all the values of $g$ are non-void. Therefore, it follows from the proposition that $g(c)=\{u\}$ for a $u \in X$. 
Then $g(u)=g^{2}(c)=f(c)$, whence, by $(\mathrm{v})$, we have $\# g(u)>1$, implying that $u \neq c$. On account of $(\mathrm{v})$ the set $g(u)$ contains a point $v \in X \backslash\{c\}$. Moreover, according to (vi), $c \in f(c)=g(u)$. Therefore, since

$$
g(v) \cup g(c) \subset g(g(u))=f(u),
$$

(i) gives $g(v)=g(c)$. Consequently, $f(v)=f(c)$, which contradicts (i) and (v).

\section{Examples}

1. Consider $f:[0,1] \rightarrow 2^{[0,1]}$ given by

$$
f(x)= \begin{cases}\frac{3}{2} x, & \text { if } x \in\left[0, \frac{1}{2}\right) \\ {\left[\frac{1}{2}, \frac{3}{4}\right],} & \text { if } x=\frac{1}{2} \\ x, & \text { if } x \in\left(\frac{1}{2}, 1\right] .\end{cases}
$$

Then assumptions (i)-(iv) in Theorem 1 are satisfied with $c=1 / 2$ and $n=2$. Consequently, $f$ has no square root.

2. There are some properties, like e.g. strict monotonicity, continuity, lack of fixed points of $f$ in the interior of its interval domain, which guarantee the existence of iterative roots of single-valued functions (cf., e.g., [12, Chap. XV] and [13, Chap. 11]). For setvalued functions the situation is more complicated, which can be seen by considering $f_{1}:[0,1] \rightarrow 2^{[0,1]}$ and $f_{2}:[0,1] \rightarrow 2^{[0,1]}$ defined by

$$
\begin{aligned}
& f_{1}(x)= \begin{cases}\frac{5}{3} x, & \text { if } x \in\left[0, \frac{1}{2}\right), \\
{\left[\frac{2}{3}, \frac{5}{6}\right],} & \text { if } x=\frac{1}{2}, \\
\frac{2}{3}(x-1)+1, & \text { if } x \in\left(\frac{1}{2}, 1\right],\end{cases} \\
& f_{2}(x)= \begin{cases}\frac{4}{3} x, & \text { if } x \in\left[0, \frac{1}{2}\right), \\
{\left[\frac{2}{3}, \frac{5}{6}\right],} & \text { if } x=\frac{1}{2}, \\
\frac{1}{3}(x-1)+1, & \text { if } x \in\left(\frac{1}{2}, 1\right],\end{cases}
\end{aligned}
$$

respectively. Both of them are upper semicontinuous and have no fixed points in $(0,1)$. Moreover, $\left.f_{1}\right|_{[0,1 / 2)}$ and $\left.f_{1}\right|_{(1 / 2,1]}$ are both strictly increasing and the (single-valued) function $\left.f_{1}\right|_{[0,1] \backslash\{1 / 2\}}$ is continuous. For $f_{2}$ we have even more: $\left.f_{2}\right|_{[0,1] \backslash\{1 / 2\}}$ is strictly increasing and continuous. Nevertheless, by Theorem 1 , where we take $c=1 / 2$ and $n=3-j$ for $f_{j}(j=1,2)$, both $f_{1}$ and $f_{2}$ have no square roots. Observe also that $1 / 2 \notin f_{1}(1 / 2)$ and $1 / 2 \notin f_{2}(1 / 2)$, that is, condition (vi) is not satisfied. Consequently, Theorem 1 does not follow from Theorem 2 .

3. In the case of $f_{3}$ as shown in Fig. 5 we have no roots again, as observed for $n=4$.

4. The shape of the graph of $f_{4}$ (see Fig. 6), given by

$$
f_{4}(x)= \begin{cases}\frac{1}{2} x, & \text { if } x \in\left[0, \frac{1}{2}\right), \\ {\left[\frac{1}{4}, \frac{3}{4}\right],} & \text { if } x=\frac{1}{2}, \\ \frac{1}{2}(x-1)+1, & \text { if } x \in\left(\frac{1}{2}, 1\right],\end{cases}
$$




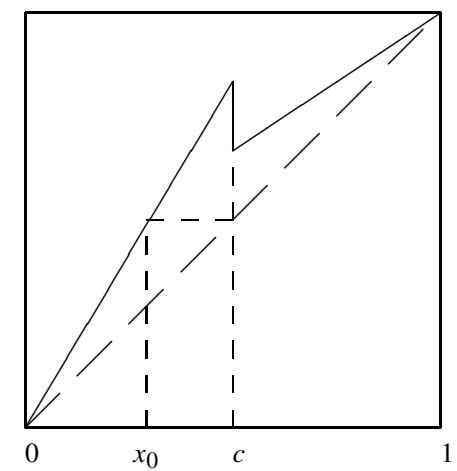

Fig. 3: $f_{1}$ with $n=2$

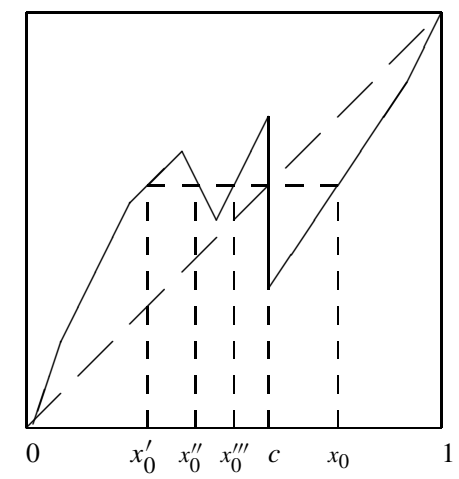

Fig. 5: $f_{3}$ with $n=4$

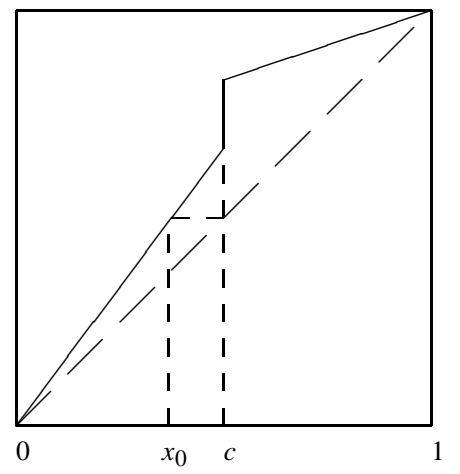

Fig. 4: $f_{2}$ with $n=1$

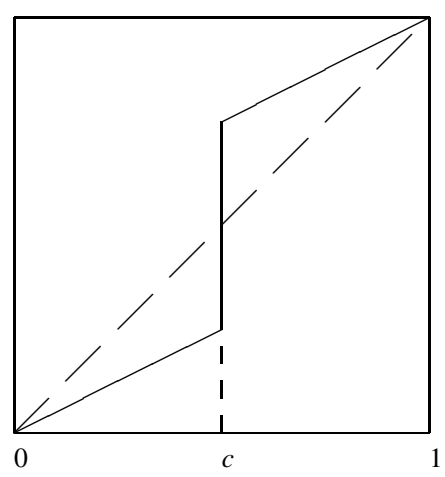

Fig. 6: $f_{4}$ has roots

is similar to the graph of $f_{2}$, but $f_{4}$ has a square root. One can easily verify that $g$ : $[0,1] \rightarrow 2^{[0,1]}$, defined by

$$
g(x)= \begin{cases}\frac{1}{\sqrt{2}} x, & \text { if } x \in\left[0, \frac{1}{2}\right), \\ {\left[\frac{1}{2 \sqrt{2}}, 1-\frac{1}{2 \sqrt{2}}\right],} & \text { if } x=\frac{1}{2}, \\ \frac{1}{\sqrt{2}}(x-1)+1, & \text { if } x \in\left(\frac{1}{2}, 1\right],\end{cases}
$$

satisfies $g^{2}=f$. Observe, however, that Condition (ii) fails, where $c$ has to be $1 / 2$.

5. Consider the set-valued functions $f_{5}:[0,1] \rightarrow 2^{[0,1]}$ and $f_{6}:[0,1] \rightarrow 2^{[0,1]}$ defined by

$$
f_{5}(x)= \begin{cases}\frac{3}{2} x, & \text { if } x \in\left[0, \frac{1}{2}\right), \\ \left\{\frac{1}{2}, \frac{3}{4}\right\}, & \text { if } x=\frac{1}{2}, \\ x, & \text { if } x \in\left(\frac{1}{2}, 1\right]\end{cases}
$$




$$
f_{6}(x)= \begin{cases}\frac{3}{2} x, & \text { if } x \in\left[0, \frac{1}{2}\right), \\ {\left[\frac{1}{2}, \frac{3}{4}\right],} & \text { if } x=\frac{1}{2}, \\ \frac{1}{2}, & \text { if } x \in\left(\frac{1}{2}, \frac{3}{4}\right], \\ 2(x-1)+1, & \text { if } x \in\left(\frac{3}{4}, 1\right],\end{cases}
$$

respectively. Condition (iii) is not satisfied by $f_{5}$ since $c=1 / 2, n=2$ and $\# f_{5}(c)=2$. For $f_{6}$ condition (iii) is not satisfied because $c=1 / 2, n=\aleph_{0}$ and $\# f_{6}(c)=\aleph_{0}$. However, they both satisfy (v) and (vi). Theorem 2 shows that none of them has a square root. Consequently, this also implies that Theorem 2 does not follow from Theorem 1.

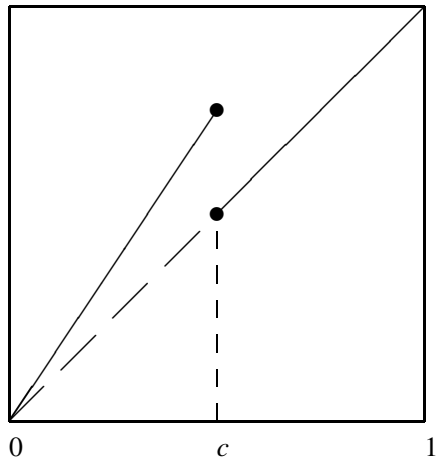

Fig. 7: $f_{5}$ with $n=2$

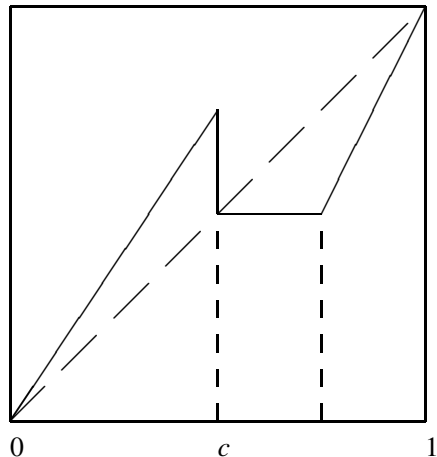

Fig. 8: $f_{6}$ with $n=\infty$

\section{Acknowledgment}

The work was supported by the University of Zielona Góra, NSFC\#10471101, NOYF-B\#10428104 and SRFDP\#20050610003.

\section{References}

[1] Babbage, Ch.: Essay towards the calculus of functions. Philosoph. Transact. (1815), 389-423; Essay towards the calculus of functions, II. Ibid. (1816), 179-256.

[2] Baron, K.: Recent results in the theory of functional equations in a single variable. Seminar LV 15 (2003), 1-16, http://www.mathematik.uni-karlsruhe.de/ ${ }^{\text {semlv }}$

[3] Baron, K.; Jarczyk, W.: Recent results on functional equations in a single variable, perspectives and open problems. Aequationes Math. 61 (2001), 1-48.

[4] Blokh, A.M.: The set of all iterates is nowhere dense in $C([0,1],[0,1])$. Trans. Amer. Math. Soc. 333 (1992), 787-798.

[5] Blokh, A.M.; Coven, E.; Misiurewicz, M.; Nitecki, Z.: Roots of continuous piecewise monotone maps of an interval. Acta Math. Univ. Comenian. (N.S.) 60 (1991), 3-10.

[6] Bogatyi, S.: On the nonexistence of iterative roots. Topology Appl. (1997), 97-123.

[7] Bronshtein, E.M.: On an iterative square root of a quadratic trinomial [Russian]. Geometric problems in the theory of functions and sets [Russian]. 24-27, Kalinin. Gos. Univ., Kalinin 1989.

[8] Choczewski, B.; Kuczma, M.: On iterative roots of polynomials. European Conference on Iteration Theory (Lisbon, 1991), 59-67, World Sci. Publishing, Singapore 1992. 
[9] Humke, P.D.; Laczkovich, M.: The Borel structure of iterates of continuous functions. Proc. Edinburgh Math. Soc. (2) 32 (1989), 483-493.

[10] Isaacs, R.: Iterates of fractional order. Canad. J. Math. 2 (1950), 409-416.

[11] Jarczyk, W.; Powierża, T.: On the smallest set-valued iterative roots of bijections. Dynamical Systems and Functional Equations (Murcia, 2000). Internat. J. Bifur. Chaos Appl. Sci. Engrg. 13 (2003), 1889-1893.

[12] Kuczma, M.: Functional equations in a single variable. Monografie Mat. 46, Polish Scientific Publishers, Warszawa 1968.

[13] Kuczma, M.; Choczewski, B.; Ger, R.: Iterative functional equations. Encyclopedia of Mathematics and Its Applications 32, Cambridge University Press, Cambridge 1990.

[14] Powierża, T.: Strong set-valued iterative roots. Grazer Math. Ber. 344 (2001), 51-56.

[15] Powierża, T.: Higher order set-valued iterative roots of bijections. Publ. Math. Debrecen 61 (2002), 315324.

[16] Rice, R.E.; Schweizer, B.; Sklar, A.: When is $f(f(z))=a z^{2}+b z+c$ ? Amer. Math. Monthly 87 (1980), 252-263.

[17] Riggert, G.: n-te iterative Wurzeln von beliebigen Abbildungen. Aequationes Math. 14 (1976), 208.

[18] Simon, K.: Some dual statements concerning Wiener measure and Baire category. Proc. Amer. Math. Soc. 106 (1989), 455-463.

[19] Targonski, Gy.: Topics in iteration theory. Vandenhoeck and Ruprecht, Göttingen 1981.

[20] Zhang, W.: A generic property of globally smooth iterative roots. Sci. China Ser. A 38 (1995), 267-272.

[21] Zhang, W.: PM functions, their characteristic intervals and iterative roots. Ann. Polon. Math. 65 (1997), $119-128$.

[22] Zimmermann, G.: Über die Existenz iterativer Wurzeln von Abbildungen. Doctoral Dissertation, Marburg/Lahn 1978

Witold Jarczyk

Faculty of Mathematics, Computer Science and Econometrics

University of Zielona Góra

Szafrana $4 \mathrm{a}$

PL-65-516, Zielona Góra, Poland

e-mail: wjarczyk@uz.zgora.pl

Weinian Zhang (Corresponding author)

Department of Mathematics

Sichuan University

Chengdu

Sichuan 610064, P.R. China

e-mail: matzwn@126.com or matzwn@sohu.com 